\title{
Reduced Nicotinamide Adenine Dinucleotide and the
}

\section{Reduction of Oxidized Glutathione in Human Erythrocytes}

\author{
Egmond E. Rieber, Nechama S. Kosower, and Ernst R. Jaffé \\ From the Department of Medicine, Albert Einstein College of Medicine- \\ Bronx Municipal Hospital Center, New York
}

A в S T R A C T The ability of reduced nicotinamide adenine dinucleotide (NADH), generated through the activity of lactic acid dehydrogenase, to support the reduction of endogenous oxidized glutathione in intact human erythrocytes and in hemolysates was investigated. Rapid initial oxidation of endogenous reduced glutathione was effected with methyl phenylazoformate. Freshly obtained normal erythrocytes and erythrocytes deficient in glucose6-phosphate dehydrogenase activity were unable to regenerate reduced glutathione upon incubation with lactate. Only normal erythrocytes were capable of reducing oxidized glutathione after preincubation with glucose, inosine, or a medium which promoted the synthesis of increased amounts of intracellular NAD. This regeneration of reduced glutathione could be explained by the generation of reduced nicotinamide adenine dinucleotide phosphate through the metabolism of accumulated phosphorylated intermediates of glycolysis. Hemolysates prepared from both normal erythrocytes and from erythrocytes deficient in glucose-6-phosphate dehydrogenase activity were able to reduce oxidized glutathione in the presence of added lactate and NAD. The results obtained indicated either an inability of the intact erythrocyte to utilize the

This work was presented in part at the 51st Annual Meeting of the Federation of American Societies for Experimental Biology, Chicago, 21 April 1967.

Dr. Jaffé is a Career Scientist of the Health Research Council of the City of New York (I-169). Address requests for reprints to Dr. Ernst R. Jaffé, Albert Einstein College of Medicine, Yeshiva University, Eastchester Road and Morris Park Avenue, Bronx, N. Y. 10461.

Received for publication 30 June 1967 and in revised form 7 Scptember 1967.
NAD at the concentrations attained or an altered behavior of the system for the regeneration of reduced glutathione after lysis of the cell.

\section{INTRODUCTION}

The concentration of reduced glutathione (GSH) is maintained at a fairly constant level in normal human erythrocytes. Erythrocytes deficient in glucose-6-phosphate dehydrogenase (G-6-PD) activity and, therefore, possessing only a limited capacity for the generation of NADPH, are unable to maintain GSH in the reduced form upon exposure to various oxidizing agents (1). NADPH has been shown to serve as the hydrogen donor for the reduction of oxidized glutathione (GSSG) to GSH in crude or partially purified preparations of hemolysates, mammalian liver, and yeast (2-6). Under certain conditions, some of these preparations are able to utilize NADH for the reduction of GSSG $(2-4,6)$. The possible role of NADH in the reduction of GSSG in intact erythrocytes has not been clearly defined, in part because of the lack of suitable means for obtaining an initial high endogenous GSSG content. Methyl phenylazoformate (azoester) ${ }^{1}$ rapidly oxidizes the GSH of erythrocytes (7). Intact, normal human erythrocytes can regenerate GSH after treatment with azoester when incubated with glucose which permits the generation of NADPH, but erythrocytes deficient in G-6-PD activity regenerate little if any

\footnotetext{
${ }^{1}$ Methyl phenylazoformate was kindly supplied by Professor E. M. Kosower and Dr. P. K. C. Huang, Department of Chemistry, State University of New York, Stony Brook, N. Y.
} 
GSH under these experimental conditions (8). The availability of azoester has permitted an evaluation of the role of NADH in the reduction of endogenous GSSG in intact erythrocytes and in hemolysates. The present investigation has demonstrated a difference between intact erythrocytes and hemolysates in the ability to utilize a NADH generating system for the reduction of GSSG.

\section{METHODS}

Preparation and incubation of erythrocyte suspensions and hemolysates

Blood samples, anticoagulated with heparin $(2 \mathrm{mg} / 10$ $\mathrm{ml}$ blood), were obtained from six normal individuals of Caucasian descent and from one Caucasian and three Negro males whose erythrocytes had been found to be deficient in G-6-PD activity. All donors were in good health and their packed erythrocyte volumes were greater than $35 \%$. After centrifugation in the cold, we removed the plasma and buffy coat and washed the erythrocytes three times in 10-12 volumes of $0.15 \mathrm{M}$ sodium chloride solution with centrifugation. Hemolysates were prepared by thrice freezing and thawing the washed, packed erythrocytes.

Suspensions of intact or hemolyzed erythrocytes were prepared in a solution containing $0.055 \mathrm{~m}$ sodium chloride, $0.03 \mathrm{M}$ sodium phosphate, and $0.1 \mathrm{M}$ glycylglycine buffer, $\mathrm{pH} 7.4$, with or without added substrates. Oxidation of $\mathrm{GSH}$ was achieved by adding a solution of azoester to the suspensions which were kept in ice, as described previously (7), except that the azoester was dissolved directly in buffer $(5 \mu \mathrm{moles} / \mathrm{ml})$. Control mixtures were prepared in the same manner, but only buffer without azoester was added. The incubation mixtures with a total volume of 3.0-3.2 $\mathrm{ml}$ had the following composition: erythrocyte suspensions with packed cell volume $24 \pm 4 \%$ with or without $1.17 \times 10^{-3} \mathrm{M}$ azoester; hemolysates with hemoglobin concentrations $7.8 \pm 1.2 \mathrm{~g} / 100 \mathrm{ml}, 3.3 \times 10^{-3} \mathrm{M}$ magnesium chloride, $2.12 \times 10^{-3} \mathrm{M}$ adenosine triphosphate 2 (ATP) and with or without $1.17 \times 10^{-3} \mathrm{M}$ a\%oester. lactate and, with hemolysates, NAI) 2 were added in the concentrations indicated in the figures and tables. Sodium lactate was prepared from $\mathrm{L}(+)$-calcium lactate ${ }^{3}$ with an equimolar amount of sodium carbonate and was adjusted to $\mathrm{pH} 7$ with hydrochloric acid or from purified lactic acid 4 with an equimolar amount of sodium hydroxide. The initial $\mathrm{pH}$ of the incubation mixtures was between 7.15 and 7.30 .

The incubation mixtures, kept in ice for about $10 \mathrm{~min}$ after the addition of azoester, were incubated at $37^{\circ} \mathrm{C}$ in air in a waterbath with occasional mixing. Aliquots of 0.2 or $0.3 \mathrm{ml}$ were removed for the determination of $\mathrm{GSH}$ before and after incubation for 30 and $60 \mathrm{~min}$.

Preparation of erythrocytes with increased concentrations of NAD

Erythrocytes with increased concentrations of NAD were prepared by incubating suspensions of washed cells (packed cell volume 66-68\%) with $5.8 \times 10^{-2} \mathrm{M}$ glucose, $9.2 \times 10^{-3} \mathrm{M}$ L-glutamine, $2.3 \times 10^{-2} \mathrm{M}$ inorganic phosphate, $1.38 \times 10^{-4} \mathrm{M}$ nicotinic acid, penicillin, $0.6 \mathrm{mg} / \mathrm{ml}$, and streptomycin, $0.6 \mathrm{mg} / \mathrm{ml}$, as described previously (9). We prepared control suspensions by incubating erythrocytes in a medium in which the nicotinic acid was replaced by an equimolar amount of nicotinamide. When inosine replaced glucose, it was added in an anvunt equivalent to the concentration of glucose. After incubation for 8 or $16 \mathrm{hr}$, we washed the erythrocytes three times in the cold with $0.15 \mathrm{M}$ sodium chloride solution with centrifugation. Incubation mixtures of these prein-

2 P.L. Biochemicals, Milwaukee, Wis.

${ }^{3}$ Calbiochem, Los Angeles, Calif.

4 Sigma Chemical Corporation, St. Louis, Mo.

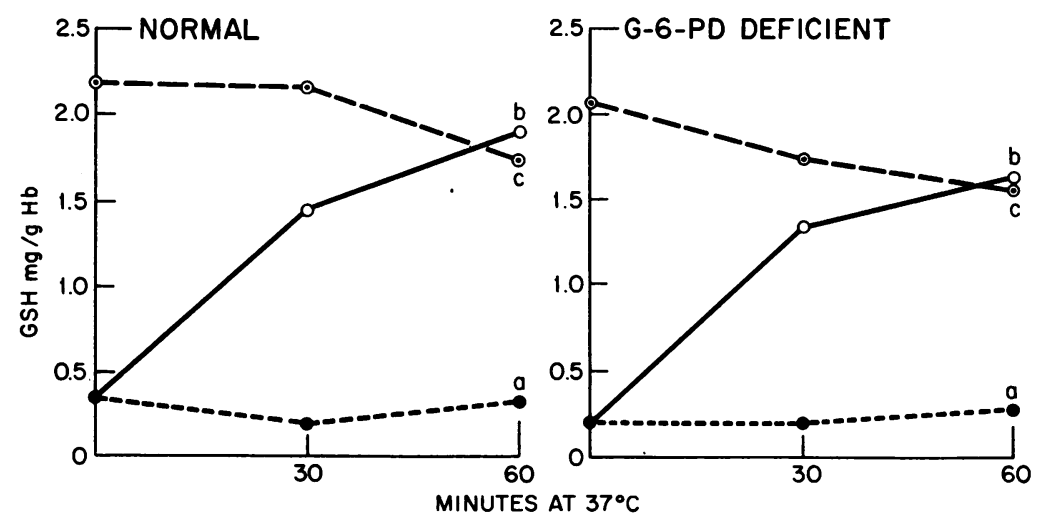

FIGURE 1 Regeneration of reduced glutathione (GSH) in hemolysates. $a$, Azoester-treated hemolysates incubated in the absence of lactate. $b$, Azoestertreated hemolysates incubated with added lactate. $c$, Control hemolysates not treated with azoester incubated with added lactate. Final concentrations: $1.4 \times$ $10^{-2} \mathrm{M}$ sodium lactate; $1.36 \times 10^{-3} \mathrm{M}$ NAD. Other additives as described in text. 
TABLE I

Effect of NAD Concentration on Reduced Glutathione (GSH) Regeneration in Hemolysates*

\begin{tabular}{|c|c|c|c|c|c|}
\hline \multirow[b]{2}{*}{ Hemolysate } & \multirow[b]{2}{*}{ NAD } & \multicolumn{2}{|c|}{$\begin{array}{c}\text { Hemolysates of } \\
\text { normal RBC }\end{array}$} & \multicolumn{2}{|c|}{$\begin{array}{c}\text { Hemolysates of } \\
\text { RBC deficient } \\
\text { in G-6-PD } \\
\text { activity }\end{array}$} \\
\hline & & 0 Time & $60 \mathrm{Min}$ & 0 Time & $60 \mathrm{Min}$ \\
\hline & Amoles $/ \mathrm{ml}$ & & GSH $m$ & $\mathrm{~g} / \mathrm{g} \mathrm{Hb}$ & \\
\hline \multirow[t]{6}{*}{ Azoester treated } & 0.43 & 0.25 & 0.55 & 0.16 & 0.77 \\
\hline & 0.85 & 0.23 & 0.68 & 0.15 & 1.04 \\
\hline & 1.70 & 0.25 & 1.08 & 0.16 & 1.48 \\
\hline & 3.40 & 0.20 & 1.50 & 0.15 & 1.48 \\
\hline & 6.81 & 0.26 & 1.60 & 0.13 & 1.46 \\
\hline & 13.62 & 0.28 & 1.63 & 0.16 & 1.46 \\
\hline $\begin{array}{l}\text { Azoester omitted } \\
\quad \text { (control) }\end{array}$ & 6.81 & 1.80 & 1.57 & 1.48 & 1.35 \\
\hline
\end{tabular}

* All samples contained sodium lactate $14.8 \times 10^{-2} \mathrm{M}$. Other additives as described in text.

cubated cells were then prepared with the same concentrations of buffer and azoester as described above.

\section{Analytic methods}

The concentrations of GSH were measured with 5,5'dithiobis-(2-nitrobenzoic acid) 5 as described by Beutler et al. (10). The activity of G-6-PD in hemolysates was determined by the method of Kornberg and Horecker as modified by Marks (11). The concentrations of NAD were measured by a fluorometric method with alcohol dehydrogenase in filtrates prepared after the addition of aliquots of the erythrocyte suspensions to 2 volumes of cold $7 \%$ trichloroacetic acid $(9,12)$. Concentrations of hemoglobin and packed erythrocyte volumes were determined by routine hematologic methods.

\section{RESULTS}

Oxidation and regeneration of GSH in hemolysates

The addition of azoester to hemolysates resulted in a decrease in the concentration of GSH to 10 $15 \%$ of the content of control, untreated hemolysates before incubation. Incubation of azoestertreated hemolysates with lactate and NAD promoted the regeneration of GSH which attained control values within $60 \mathrm{~min}$. In the absence of lactate, no regeneration of GSH occurred. The results of typical experiments are shown in Fig. 1. Hemolysates prepared from normal erythrocytes and from erythrocytes deficient in G-6-PD activity behaved similarly. There was no difference in the behavior of enzyme-deficient hemolysates prepared from erythrocytes of Negro or Caucasian indi-

5 Calbiochem, Los Angeles, Calif. viduals. Thus, hemolysates were capable of reducing endogenous GSSG to GSH, regardless of the level of activity of G-6-PD in the hemolysates.

The extent of regeneration of GSH was clcpendent upon the concentrations of NAD and lactate in the incubation mixtures. Increasing the concentration of added NAD permitted the regeneration of increasing amounts of GSH in hemolysates of both normal erythrocytes and erythrocytes deficient in G-6-PD activity (Table I). Variability in the amount of NAD required for maximal regeneration of GSH was noted. The dependency of regeneration of GSH on the concentration of lactate was demonstrated by the data in Table II.

A progressive increase in the concentration of GSH occurred during $60 \mathrm{~min}$ of incubation (Fig. 1 ), but continuing the incubation for more than $1 \mathrm{hr}$ did not lead to further increases. Omission of ATP from the incubation mixture did not alter the rate of regeneration of GSH. No difference in the regeneration of GSH was observed during incubation for $60 \mathrm{~min}$ when the initial $\mathrm{pH}$ of the hemolysate mixtures was varied between 6.96 and 7.85 by the addition of hydrochloric acid or sodium hydroxide. A decrease of about $10-25 \%$ in the content of GSH occurred during incubation of control, untreated hemolysates for $60 \mathrm{~min}$ (Fig. 1, curves $c$ ). This decrease was not influenced by altering the concentrations of NAD, lactate, or ATP and occurred with hemolysates prepared from both normal erythrocytes and erythrocytes deficient in G-6-PD activity.

TABLE II

Effect of Sodium Lactate Concentration on Reduced Glutathione (GSH) Regeneration in Hemolysates*

\begin{tabular}{cccc}
\hline Na lactate & 0 Time & 30 Min & $60 \mathrm{Min}$ \\
\hline$\mu$ moles $/ \mathrm{ml}$ & & G.SH $\mathrm{mg} / \mathrm{g} \mathrm{Hb}$ & \\
4.61 & 0.26 & 0.59 & 0.87 \\
9.22 & 0.30 & 0.69 & 1.03 \\
13.83 & 0.15 & 0.75 & 1.45 \\
18.44 & 0.26 & 1.14 & 1.62 \\
23.05 & 0.29 & 1.43 & 1.72 \\
\hline
\end{tabular}

* Hemolysate of normal erythrocytes treated with azoester. All samples contained $1.70 \times 10^{-3} \mathrm{M}$ NAD. GSH concentration before the addition of azoester was $2.08 \mathrm{mg} / \mathrm{g}$ $\mathrm{Hb}$. Other additives as described in text. 


\section{Oxidation and regeneration of GSH in intact erythrocytes}

Freshly prepared erythrocyte suspensions. The concentration of GSH decreased to $6-7 \mathrm{mg} / 100 \mathrm{ml}$ of erythrocytes after the addition of azoester. No regeneration of GSH was observed when azoestertreated normal erythrocytes or erythrocytes deficient in G-6-PD activity were incubated with the concentration of lactate that did promote regeneration in hemolysates (Table III). Under comparable conditions, the addition of glucose to the incubation medium promoted regeneration of GSH in normal erythrocytes but not in erythrocytes deficient in G-6-PD activity (8).

Suspensions of preincubated erythrocytes. Since regeneration of GSH in hemolysates was dependent upon the addition of NAD, as well as on lactate, we prepared erythrocytes containing increased concentrations of NAD by incubating cells with nicotinic acid and the medium described above $(9,13)$. Both normal erythrocytes and
TABLE III

Effect of Sodium Lactate on Reduced Glutathione (GSH) Regeneration in Intact Erythrocytes*

\begin{tabular}{|c|c|c|c|c|}
\hline Erythrocytes & $\begin{array}{l}\text { Azo- } \\
\text { ester }\end{array}$ & $\begin{array}{c}0 \\
\text { Time }\end{array}$ & $\begin{array}{c}30 \\
\text { Min }\end{array}$ & $\begin{array}{c}60 \\
\text { Min }\end{array}$ \\
\hline & & \multicolumn{3}{|c|}{ GSH $\mathrm{mg} / 100 \mathrm{ml} \mathrm{RBC}$} \\
\hline \multirow{2}{*}{ Normal } & + & 7 & 8 & 7 \\
\hline & - & 67 & & 66 \\
\hline \multirow[t]{2}{*}{ G-6-PD Deficient } & + & 6 & 6 & 7 \\
\hline & - & 60 & & 54 \\
\hline
\end{tabular}

* All samples contained $14.1 \times 10^{-8} \mathrm{M}$ sodium lactate. Other additives as described in text.

erythrocytes deficient in G-6-PD activity contained two to four times the concentrations of NAD present in control erythrocytes after incubation for 8 or $16 \mathrm{hr}$. When nicotinic acid was replaced by an equimolar amount of nicotinamide, when glucose was replaced by an equimolar amount of inosine, or when the cells were preincubated with

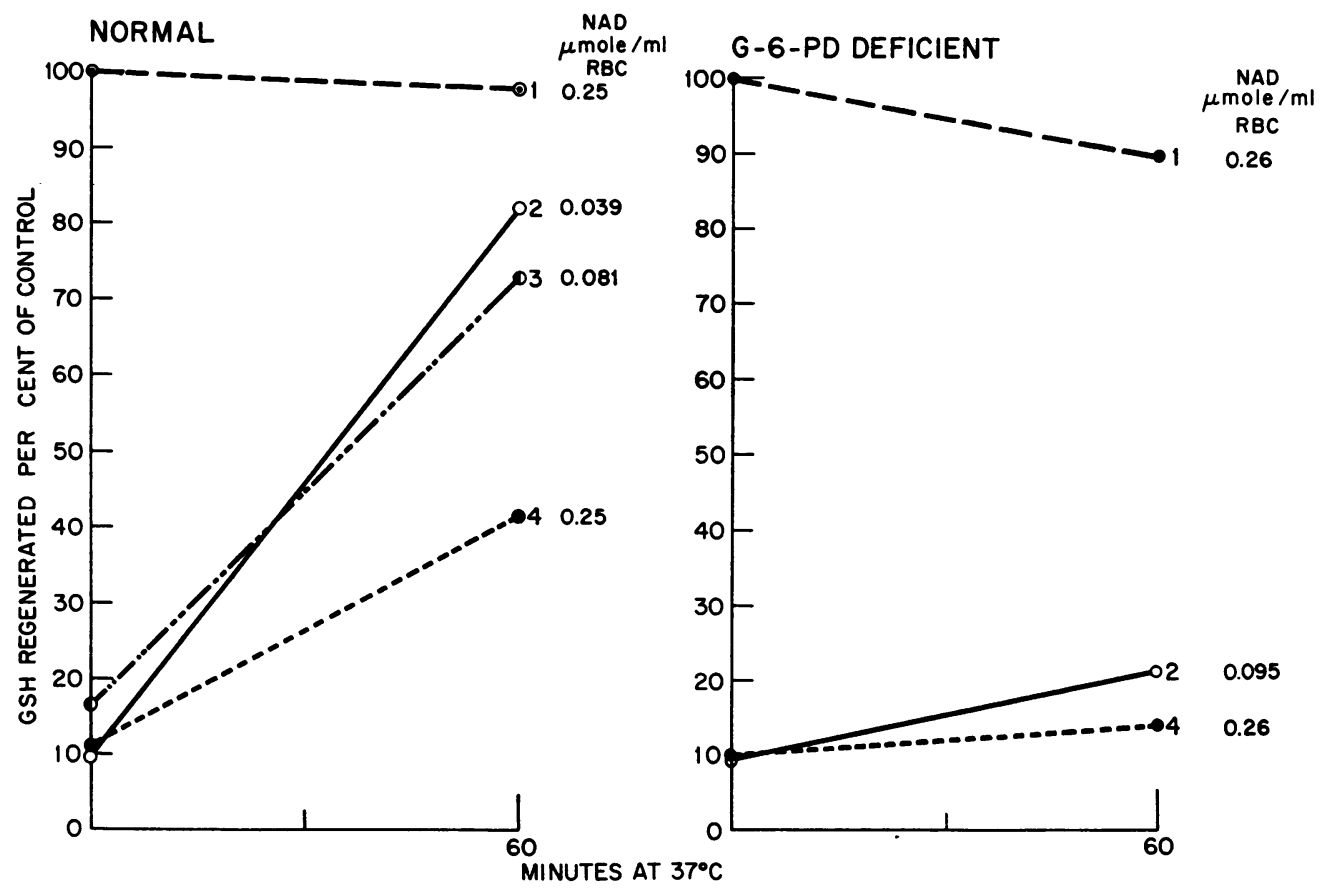

FIGURE 2 NAD content and regeneration of reduced glutathione (GSH) in intact human erythrocytes. 1 , Control erythrocytes not treated with azoester, preincubated with complete NAD enrichment medium. 2, Azoester-treated erythrocytes, inosine substituted for glucose in enrichment medium. 3, Azoestertreated erythrocytes, preincubated with glucose alone. 4, Azoester-treated erythrocytes preincubated with complete NAD enrichment medium. Final concentration of sodium lactate in all samples $2.8 \times$ $10^{-2} \mathrm{M}$. Other additives as described in text. Control values for GSH $64 \mathrm{mg} \pm 4 / 100 \mathrm{ml}$ erythrocytes $=$ $100 \%$. 
glucose and inorganic phosphate alone, elevated NAD concentrations were not observed.

When washed, preincubated erythrocytes were treated with azoester, the concentration of GSH decreased to $3-8 \mathrm{mg} / 100 \mathrm{ml}$ of erythrocytes. Upon incubation of azoester-treated preincubated erythrocytes with added lactate, a completely different pattern of regeneration of GSH was observed when normal erythrocytes were compared with erythrocytes deficient in G-6-PD activity (Fig. 2). Normal erythrocytes were capable of reducing endogenous GSSG regardless of the concentration of NAD. Actually, more GSH was regenerated in erythrocytes preincubated with glucose alone or inosine where the concentration of NAD was not elevated (Fig. 2, left, curves 2 and 3). GSH was regenerated to the extent of about $45 \%$ of the concentration in the control, untreated erythrocyte suspension when the NAD concentration was about $0.3 \mu \mathrm{mole} / \mathrm{ml}$ of cells. $69-82 \%$ of the control level was attained in suspensions with NAD concentrations of about $0.1 \mu \mathrm{mole} / \mathrm{ml}$ of cells. Erythrocytes deficient in G-6-PD activity were unable to regenerate GSH under the same experimental conditions.

\section{DISCUSSION}

These studies have demonstrated that $\mathrm{NADH}$, generated through the activity of lactic acid dehydrogenase upon the conversion of lactate to pyruvate, was able to serve as the hydrogen donor for reduction of endogenous GSSG to GSH in hemolysates of normal human erythrocytes and erythrocytes deficient in G-6-PD activity. These results were consistent with previous observations on the reduction of exogenous GSSG by hemolysates of human erythrocytes when NADH was added $(3,4)$. Beutler and Yeh (6) suggested that the glucose-6-phosphate-6-phosphogluconic acid system was thermodynamically more suitable for the reduction of GSSG to GSH than was the lactate-pyruvate system. The lactate-pyruvate system, however, was capable of promoting the reduction of endogenous GSSG in hemolysates with normal and decreased levels of activity of G-6-PD.

Previous investigators have reported the inahility of intact, normal human erythrocytes to utilize lactate for the regeneration of GSH (6). Acetyl- phenylhydrazine, nitrite, or prolonged vigorous oxygenation of substrate-depleted erythrocytes was used to oxidize a portion of the intracellular GSH $(6,14)$. After such treatment, complete regeneration of GSH was not observed even with glucose as substrate (6). The continuous presence of oxidants and the prolonged period of incubation required for the oxidation of GSH may have led to further breakdown of GSSG or to irreversible inactivation of essential enzymes. Treatment with azoester, which has been shown to cause a rapid and stoichiometric oxidation of GSH and to permit rapid and efficient regeneration of GSH in the presence of glucose (7) was, therefore, employed in the present investigation.

Although lactate was able to support regeneration of GSH in hemolysates, no such effect was observed with intact fresh erythrocytes. Even when the intracellular content of NAD was increased in erythrocytes deficient in G-6-PD activity, no enhanced regeneration of GSH occurred with added lactate. On the other hand, GSH was regenerated in normal erythrocytes which had been preincubated with glucose, inosine, or the medium which resulted in accumulation of increased amounts of intracellular NAD. This regeneration of GSH could be adequately explained by the further metabolism of the intermediates of glycolysis which had accumulated during the period of preincubation $(15,16)$. The metabolism of these phosphorylated intermediates through the normally functioning hexose monophosphate shunt pathway would lead to the generation of NADPH for the reduction of GSSG to GSH. This hypothesis would be consistent with the observation that more GSH was regenerated under conditions where intermediates were not utilized for NAD synthesis. Inosine whose metabolism has been reported to lead to the accumulation of large amounts of triose phosphate and hexose diphosphate $(16,17)$ also has been shown to inhibit the synthesis of NAD, presumably because of competition between nicotinic acid and hypoxanthine for the available phosphoribosylpyrophosphate (9).

The failure of erythrocytes deficient in G-6-PD activity to regenerate GSH upon incubation with lactate is compatible with the thesis that the intact human erythrocyte cannot utilize the available $\mathrm{NADH}$ as the hydrogen donor for GSSG-re- 
ductase activity. This inability to regenerate GSH in enzyme-deficient erythrocytes under conditions where normal erythrocytes can regenerate GSH also makes it unlikely that $\mathrm{NADH}$ generated through the activity of glyceraldehyde-3-phosphate dehydrogenase can support GSSG-reductase activity in the intact erythrocyte (6).

No information is available on the affinity of GSSG-reductase for NADH in the intact cell. Compartmentalization and, therefore, localized variations in subcellular concentrations of enzymes and cofactors may exist. The available concentration of NADH in the intact cell could be the ratelimiting factor in NADH-dependent GSSG reduction. The NADH-dependent reduction of GSSG achieved in lysates could also be due to differences in the ionic environment of the enzyme or to a conformational change in the enzyme with activation of previously inactive sites.

\section{ACKNOWLEDGMENT}

This work was supported by U. S. Public Health Service grants T1-AM 5435, HE-10039, and HE-10041.

\section{REFERENCES}

1. Beutler, E. 1966. Glucose-6-phosphate dehydrogenase deficiency. In The Metabolic Basis of Inherited Disease. J. B. Stanbury, J. B. Wyngaarden, and D. S. Fredrickson, editors. McGraw-Hill Book Company, New York. 2nd edition. 1060.

2. Racker, E. 1955. Glutathione reductase from bakers yeast and beef liver. J. Biol. Chem. 217: 855.

3. Francoeur, M., and O. F. Denstedt. 1954. Metabolism of mammalian erythrocytes. VII. The glutathione reductase of the mammalian erythrocyte. Can. J. Biochem. Physiol. 32: 663.

4. Carson, P., S. Schrier, and C. L. Flanagan. 1957. Use of DPNH as coenzyme for glutathione reductase of hemolysates. Federation Proc. 16: 19.
5. Scott, E. M., I. W. Duncan, and V. Ekstrand. 1963. Purification and properties of glutathione reductase of human erythrocytes. J. Biol. Chem. 238: 3928.

6. Beutler, E., and M. K. Y. Yeh. 1963. Erythrocyte glutathione reductase. Blood. 21 : 573.

7. Kosower, N. S., G. A. Vanderhoff, E. M. Kosower, and P-K. C. Huang. 1965. Decreased glutathione content of human erythrocytes produced by methyl phenylazoformate. Biochcm. Biophy's. Res. Commun. 20: 469.

8. Kosower, N. S., G. A. Vanderhoff, and I. M. London. 1967. The regeneration of reduced glutathione in normal and glucose-6-phosphate dehydrogenase deficient human red blood cells. Blood. 29: 313.

9. Jaffé, E. R., and G. Neumann. 1965. The synthesis of pyridine nucleotides in fresh and stored human erythrocytes. Transfusion. 5: 412 .

10. Beutler, E., O. Duron, and B. M. Kelly. 1963. Improved method for the determination of blood glutathione. J. Lab. Clin. Med. 61: 882 .

11. Marks, P. A. 1958. Red cell glucose-6-phosphate and 6-phosphogluconic dehydrogenases and nucleoside phosphorylase. Science. 127 : 1338.

12. Jacobson, K. B., and L. Astrachan. 1957. Specific methods for the micro-determination of pyridine nucleotides and pyridine nucleotide enzymes. Arch. Biochem. Biophys. 71: 69.

13. Preiss, J., and P. Handler. 1957. Synthesis of diphosphopyridine nucleotide from nicotinic acid by human erythrocytes in vitro. J. Am. Chem. Soc. 79: 1514.

14. Harley, J. D., and H. Robin. 1962. The effect of the nitrite ion on intact human erythrocytes. Blood. 20: 710.

15. Tsuboi, K. K., and K. Fukunaga. 1965. Inorganic phosphate and enhanced glucose degradation by the intact erythrocyte. J. Biol. Chem. 240: 2806.

16. Reinauer, H., and F. H. Bruns. 1964. Limitierende Faktoren der Glykolyse in roten Blutzellen des Menschen. Biochem. Z. 340: 503.

17. Shafer, A. W., and G. R. Bartlett. 1962. Phosphorylated carbohydrate intermediates of the human erythrocyte during storage in acid citrate dextrose. III. Effect of incubation at $37^{\circ} \mathrm{C}$ with inosine, inosine plus adenine, and adenosine after storage for 6,10 , 14, and 18 weeks. J. Clin. Invest. $41: 690$. 